\title{
Wnt/ $\beta$-catenin signaling accelerates mouse lung tumorigenesis by imposing an embryonic distal progenitor phenotype on lung epithelium
}

\author{
Eugenia C. Pacheco-Pinedo,1,2,3 Amy C. Durham, ${ }^{4}$ Kathleen M. Stewart, ${ }^{1,2}$ Ashley M. Goss, ${ }^{5}$ \\ Min Min Lu,1,2 Francesco J. DeMayo, 6 and Edward E. Morrisey1,2,3,5 \\ ${ }^{1}$ Department of Medicine, ${ }^{2}$ Cardiovascular Institute, ${ }^{3}$ nstitute for Regenerative Medicine, ${ }^{4}$ Department of Pathobiology, \\ School of Veterinary Medicine, and 5Department of Cell and Developmental Biology, University of Pennsylvania, Philadelphia, Pennsylvania, USA. \\ ${ }^{6}$ Department of Molecular and Cellular Biology, Baylor College of Medicine, Houston, Texas, USA.
}

\begin{abstract}
Although mutations in Kras are present in $21 \%$ of lung tumors, there is a high level of heterogeneity in phenotype and outcome among patients with lung cancer bearing similar mutations, suggesting that other pathways are important. Wnt/ $\beta$-catenin signaling is a known oncogenic pathway that plays a well-defined role in colon and skin cancer; however, its role in lung cancer is unclear. We have shown here that activation of Wnt/ $\beta$-catenin in the bronchiolar epithelium of the adult mouse lung does not itself promote tumor development. However, concurrent activation of $\mathrm{Wnt} / \beta$-catenin signaling and expression of a constitutively active Kras mutant (KrasG12D) led to a dramatic increase in both overall tumor number and size compared with KrasG12D alone. Activation of Wnt/ $\beta$-catenin signaling altered the Kras G12D tumor phenotype, resulting in a phenotypic switch from bronchiolar epithelium to the highly proliferative distal progenitors found in the embryonic lung. This was associated with decreased E-cadherin expression at the cell surface, which may underlie the increased metastasis of tumors with active $\mathrm{Wnt} / \beta$-catenin signaling. Together, these data suggest that activation of Wnt/ $\beta$-catenin signaling can combine with other oncogenic pathways in lung epithelium to produce a more aggressive tumor phenotype by imposing an embryonic distal progenitor phenotype and by decreasing E-cadherin expression.
\end{abstract}

\section{Introduction}

Lung cancer is the second most common cancer in both men and women, accounting for approximately $15 \%$ of all newly diagnosed cancers $(1,2)$. Despite extensive investment of research dollars into developing new treatments for lung cancer, it remains responsible for $29 \%$ of cancer deaths in the United States, which is more than breast, colon, and prostate cancer combined. The overall 5-year survival rate is only $15.8 \%$, making lung cancer one of the most deadly and difficult to diagnose cancers in humans.

Although smoking is the primary cause of most lung cancer, approximately $10 \%-15 \%$ of lung cancers are found in nonsmokers, suggesting additional genetic causes of this disease. Extensive research into the underlying molecular insults leading to lung epithelial oncogenesis has resulted in the finding that Kras and EGFR mutations account for a large number of pulmonary cancers (3-7). However, there is significant heterogeneity among patients with similar mutations, suggesting the contribution of additional molecular pathways in the regulation of lung oncogenesis.

Wnt/ $\beta$-catenin signaling is a well-described oncogenic pathway that plays important roles in several cancers, including colon cancer, where it is the most common genetic cause of oncogenesis $(8,9)$. The role of Wnt/ $\beta$-catenin signaling in lung cancer remains somewhat unclear. Mutations in $\beta$-catenin and adenomatous polyposis coli (APC), the most commonly mutated factors found in other cancers, are found only rarely in human lung tumors, and previous mouse models of Wnt $/ \beta$-catenin activation in the postnatal

Conflict of interest: The authors have declared that no conflict of interest exists. Citation for this article: J Clin Invest. 2011;121(5):1935-1945. doi:10.1172/JCI44871. lung have not produced a significant level of lung oncogenesis $(10-14)$. Recently, it has been shown using human lung tumor cell lines that increased Wnt $/ \beta$-catenin signaling can lead to increased tumor metastasis (15). These data suggest that Wnt $/ \beta$-catenin signaling may play an important role in some aspects of lung tumorigenesis, but its full role in vivo remains unclear.

In the current study, we show that activation of $\mathrm{Wnt} / \beta$-catenin signaling alone in bronchiolar epithelium does not lead to lung oncogenesis. However, activation of Wnt $/ \beta$-catenin signaling along with expression of the Kras mutant KrasG12D led to a far more aggressive lung tumor phenotype than expression of KrasG12D alone. The Kras oncogenic phenotype was altered by the activation of Wnt/ $\beta$-catenin, leading to a phenotypic switch of bronchiolar Clara cells to distal embryonic lung progenitors, which express high levels of developmental genes associated with increased proliferation and decreased differentiation. Moreover, coexpression of oncogenic Kras and Wnt/ $\beta$-catenin led to a dramatic reduction in E-cadherin, which may have led to a more metastatic phenotype. These data indicate that Wnt/ $\beta$-catenin signaling cooperates with other oncogenic pathways such as Kras to accelerate and alter the phenotype of resulting tumors, leading to a more aggressive phenotype that can have a deleterious impact on patient prognosis.

\section{Results}

Wnt/ $\beta$-catenin activation in mouse lung Clara cells is not sufficient to induce tumors but promotes carcinogenesis induced by the KrasG12D oncogene. Previous studies have shown that activation of Wnt/ $\beta$-catenin signaling in bronchiolar epithelium of the adult mouse lung does not lead to increased tumor formation or increased cell proliferation (12). However, additional studies have demonstrated a link between increased 


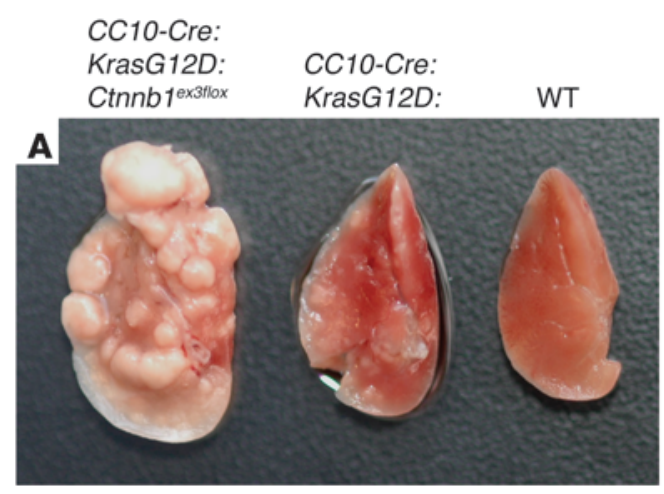

B
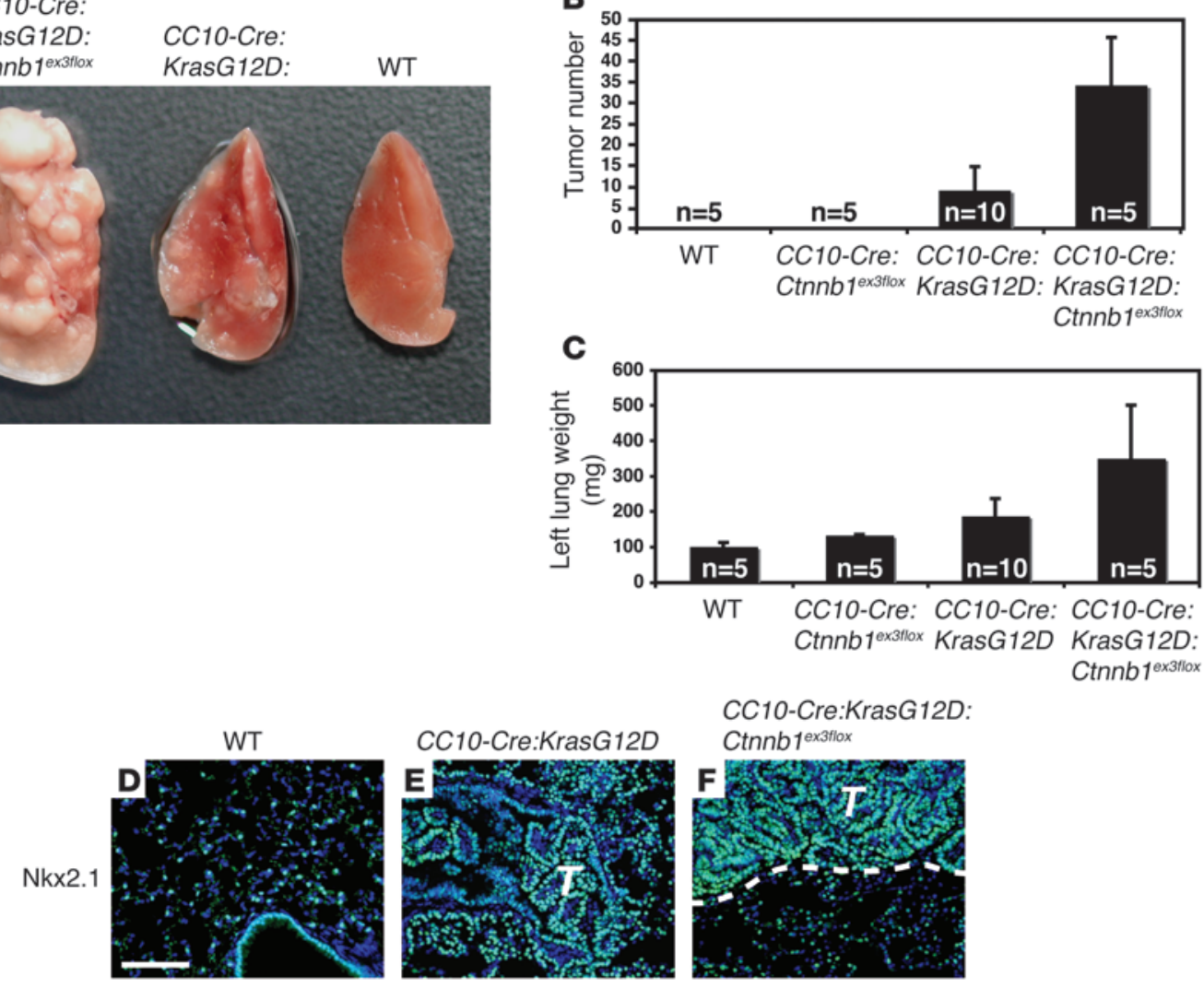

CC10-Cre:KrasG12D: Ctnnb1 exsflox $^{2}$
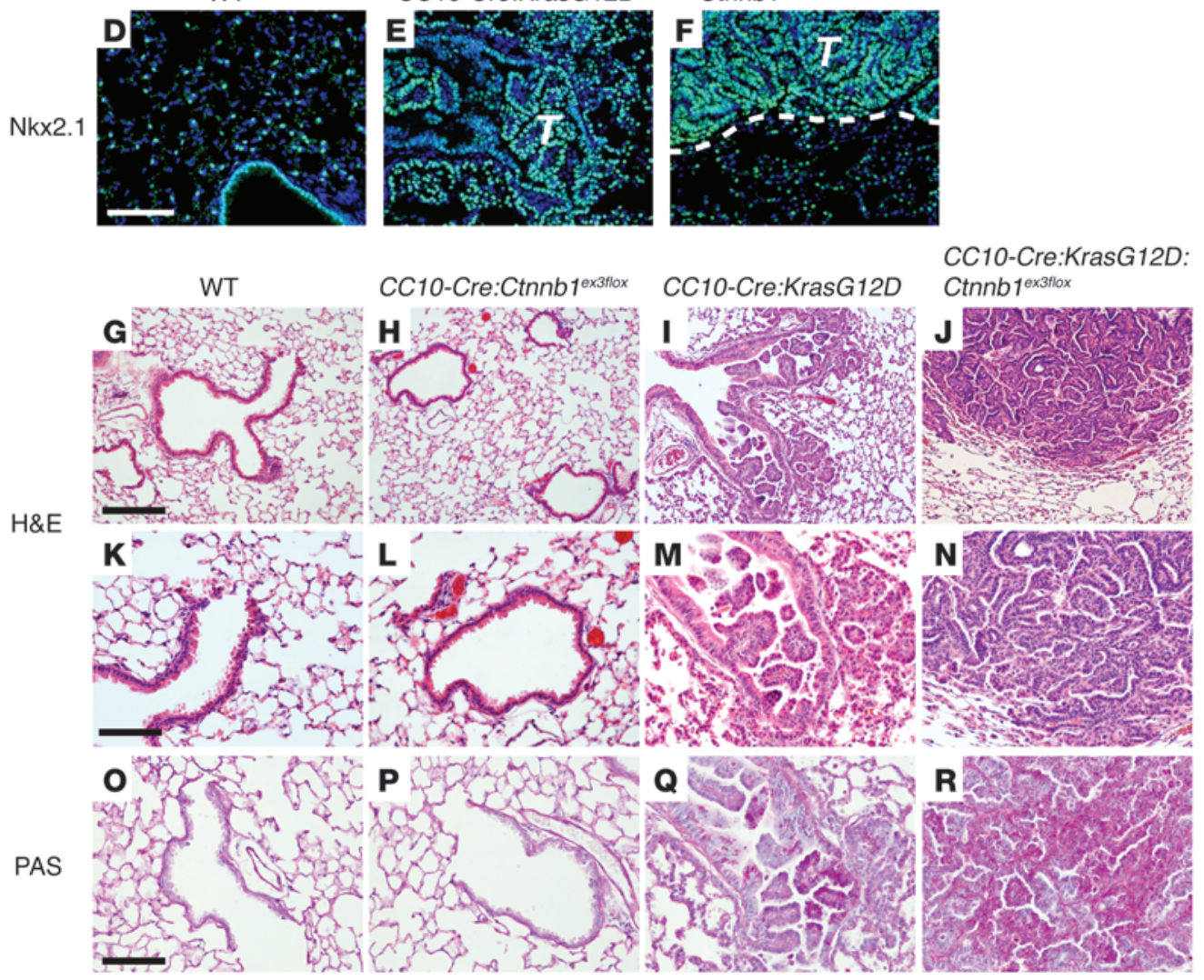

Figure 1

Coactivation of KrasG12D and Ctnnb1ex3flox leads to a synergistic increase in tumor development in the mouse lung. (A) Whole mount picture of CC10-Cre:KrasG12D:Ctnnb1ex3flox, CC10-Cre: KrasG12D, and wild-type lungs at 3 months of age. (B) Tumor numbers in lungs of mice of the indicated genotypes at 3 months of age. (C) Left lung weight from animals of the indicated genotypes at 3 months of age. Number of animals assessed in $\mathbf{B}$ and $\mathbf{C}$ is indicated in the graphs. Data in $\mathbf{B}$ and $\mathbf{C}$ are presented as mean \pm SEM. (D-F) Nkx2.1 immunostaining of lung and tumor samples of animals of the indicated genotypes at 3 months of age. (G-N) H\&E staining of histology sections from animals of the indicated genotypes at 3 months of age. Note that $\mathbf{K}-\mathbf{N}$ show higher-magnification pictures of the same samples shown in G-J. (O-R) PAS staining of histological sections from animals of the indicated genotypes at 3 months of age. Scale bars: $\mathbf{D}-\mathbf{F}, 100 \mu \mathrm{m} ; \mathbf{G}-\mathbf{J}, 400 \mu \mathrm{m} ; \mathbf{K}-\mathbf{R}, 200 \mu \mathrm{m}$. 


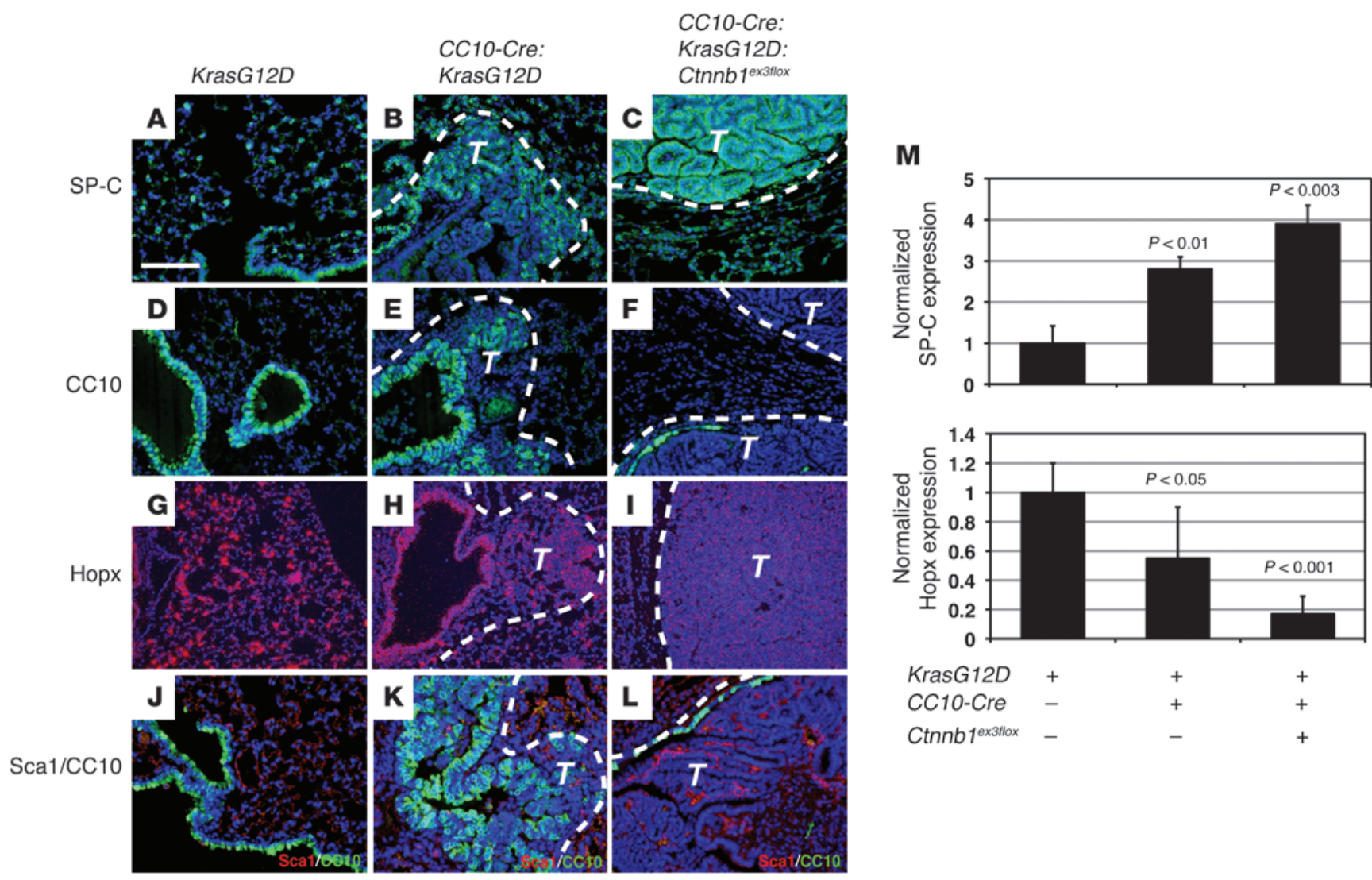

Figure 2

Bronchiolar and alveolar marker gene expression in lungs expressing KrasG12D and Ctnnb1ex3flox oncogenes. SP-C (A-C), CC10 (D-F), and Sca1/CC10 (J-L) immunostaining of lungs from animals of the indicated genotypes at 3 months of age. (G-I) In situ hybridization for expression of Hopx in lungs from animals of the indicated genotypes at 3 months of age. (M) Q-PCR for expression of SP-C and Hopx in parallel lung cDNA samples from animals of the indicated genotypes at 3 months of age. Three individual animals of the indicated genotypes were used for the Q-PCR analysis shown in $\mathbf{M}$, and the data are presented as mean \pm SEM. Scale bar: $100 \mu \mathrm{m}$.

Wnt/ $\beta$-catenin signaling and lung tumor metastasis and cell proliferation in human lung tumor cell lines $(15-17)$. To address this apparent discrepancy, we induced expression of an activated form of $\beta$-catenin by itself or in combination with the well-characterized Kras $G 12 D$ oncogenic form of Kras, which has been demonstrated to promote lung tumorigenesis $(18,19)$, specifically in postnatal bronchiolar epithelium of the lung. The Ctnnb1 $1^{\text {ex } 3 \text { flox }}$ allele of $\beta$-catenin has loxP sites surrounding exon 3 , which contains the regulatory phosphorylation sites that control $\beta$-catenin protein stability. Cremediated loss of exon 3 leads to increased $\beta$-catenin accumulation and increased Wnt signaling (20). In these experiments, we used a previously described CC10-Cre line that expresses Cre recombinase exclusively in postnatal Clara cells in the lung (21). This Cre line exhibits an intermediate rate of recombination efficiency, with $30 \%-$ $50 \%$ of Clara cells exhibiting recombination using the R26R-lacZ reporter (data not shown and ref. 21).

In agreement with previous studies (12), expression of the Ctnnb1 $1^{\text {ex } 3 f l o x}$ mutant in Clara cells did not result in an increase in lung tumor development or proliferation up to 18 months of age (Figure 1, A-C, and data not shown). After 3 months, expression of the Kras G12D mutant in Clara cells resulted in extensive formation of hyperplastic regions in the adult lung, many of which were located in the bronchioalveolar duct junction (BADJ) region (Figure 1,
A, E, I, and M). Some of these had developed into adenomas by 3 months (Figure 1, I and M). In contrast, expression of both Ctnnb1 $1^{\text {ex } 3 f l o x}$ and Kras G12D for 3-4 months resulted in a significant increase in both the number of lung tumors and lung tumor size (Figure 1, A and B). This resulted in increased net weight of the lungs (Figure 1C). To independently verify the changes in tumor number, size, and stage, we performed a blinded analysis with a pathologist who graded histological samples without knowing the genotype using previously published criteria (22). These results

Table 1

Summary of developmental genes altered in CC10-Cre:KrasG12D:Ctnnb1ex3flox tumor epithelium

\begin{tabular}{cccc} 
Transcript ID & Gene symbol & RefSeq & $\begin{array}{c}\text { Fold change } \\
\text { vs. WT }\end{array}$ \\
10530819 & Hopx & NM_175606 & -2.43681 \\
10431210 & Wnt7b & NM_009528 & 2.05218 \\
10399691 & Id2 & NM_010496 & 2.25763 \\
10382328 & Sox9 & NM_011446 & 7.80553 \\
10399421 & Mycn & NM_008709 & 3.22383 \\
\hline
\end{tabular}

RefSeq, NCBI reference sequence accession number. 


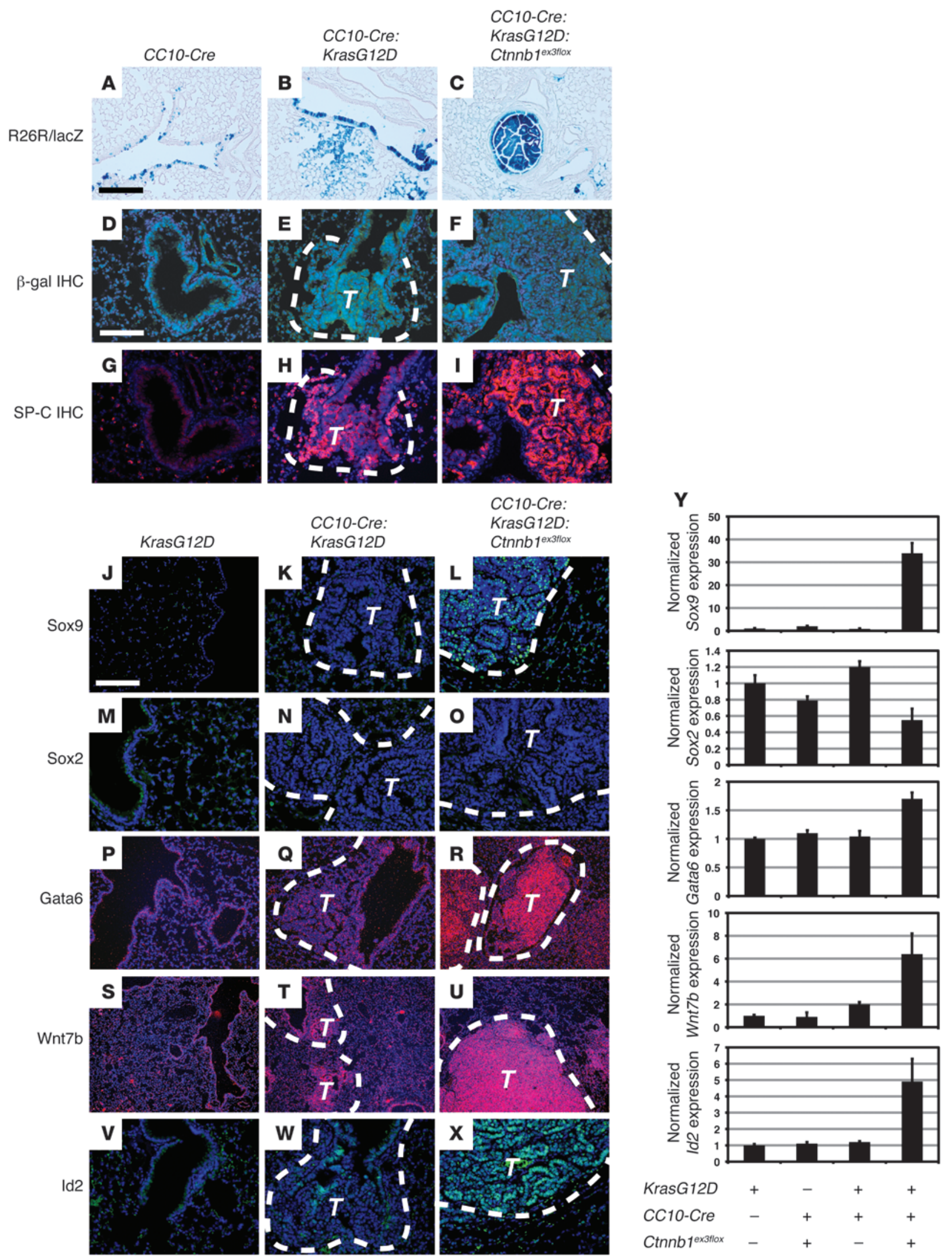




\section{Figure 3}

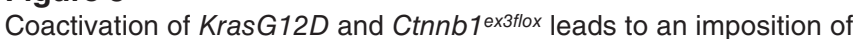
an embryonic distal progenitor phenotype on tumor epithelium derived from Clara cells of the adult lung. (A-C) $\beta$-Galactosidase staining of CC10-Cre:R26RlacZ fated cells showing that the tumors in CC10-Cre: KrasG12D and CC10-Cre:KrasG12D:Ctnnb1ex3flox animals at 3 months of age are derived from Clara cells. (D-F) Immunostaining of epithelium of CC10-Cre, CC10-Cre:KrasG12D, and CC10-Cre:KrasG12D: Ctnnb1 ex3flox mice for $\beta$-galactosidase expression at 3 months of age. (G-I) Immunostaining of epithelium of CC10-Cre, CC10-Cre:Kras-

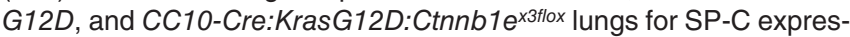
sion. Assessment of Sox9 (J-L), Sox2 (M-O), Gata6 (P-R), Wnt7b (S-U), and Id2 ( $\mathbf{V}-\mathbf{X})$ expression using immunostaining ( $\mathbf{J}-\mathbf{O}$ and $\mathbf{V}-\mathbf{X})$ or in situ hybridization $(\mathbf{P}-\mathbf{U})$ in animals of the indicated genotypes at 3 months of age. (Y) Q-PCR of these same genes. Three individual animals of the indicated genotype were used for Q-PCR analysis shown in $\mathbf{Y}$, and data are presented as mean \pm SEM. Changes in gene expression for Sox9 $(P<0.001)$, Sox2 $(P<0.05)$, Gata6 $(P<0.07)$, Wnt7b $(P<0.005)$, and $I d 2(P<0.004)$ were considered significant as indicated. Scale bars: A-C, $400 \mu \mathrm{m}$; D-X, $100 \mu \mathrm{m}$.

show that CC10-Cre:Ctnnb1 ${ }^{\text {ex3flox }}$ :LSL-KrasG12D double mutant tumors represented a more aggressive and advanced stage of development including the presence of adenocarcinomas, whereas expression of Ctnnb1 $1^{\text {ex3flox }}$ did not produce any tumors, and expression of KrasG12D generated primarily hyperplastic regions and a small number of focal adenomas after 3-4 months (Table 1). Of note, most of the CC10-Cre:Ctnnb1 ${ }^{\text {ex3 fllox }}$ :LSL-KrasG12D double mutants died before 6 months of age due to respiratory distress (data not shown). Tumors from both the CC10-Cre:KrasG12D and CC10-Cre:Ctnnb1 ${ }^{\text {ex3flox }}$ :LSL-KrasG12D double mutants were lung epithelial in origin, as indicated by expression of the transcription factor Nkx2.1 (Figure 1, D-F). Tumors in the CC10-Cre:Ctnnb1 ${ }^{\text {ex3 } 3 \text { flox: }}$ LSL-Kras G12D double mutants also expressed higher mucus levels, as indicated by increased PAS staining, which correlates with progression to the adenocarcinoma stage, in comparison to the CC10Cre:KrasG12D single mutants (Figure 1, O-R). Expression of the mesenchymal marker vimentin was not changed between controls and the CC10-Cre:Ctnnb1 ${ }^{\text {ex3flox: }}$ LSL-Kras G12D double mutants, suggesting no increase in epithelial-mesenchymal transition caused by coexpression of oncogenic Kras and activated $\beta$-catenin (Supplemental Figure 1; supplemental material available online with this article; doi:10.1172/JCI44871DS1). Together these data indicate that coexpression of the Ctnnb1 $1^{\text {ex } 3 f l o x}$ and KrasG12D oncogenes leads to an acceleration of lung tumorigenesis, which differs significantly from expression of Ctnnb1 $1^{\text {ex } 3 f l o x}$ or Kras G12D alone.

Coexpression of Ctnnb1 $1^{\text {ex } 3 f l o x}$ and Kras G12D alters the phenotype of bronchiolar epithelial cells of the lung. To further assess the phenotype of the lung tumors resulting from coexpression of Ctnnb1 ex3flox and KrasG12D oncogenes, we performed immunostaining for the lung epithelial markers SP-C, SP-B, CC10, and Hopx, which represent different lung epithelial lineages and stages of development. SP-C and SP-B are surfactant proteins expressed primarily in alveolar epithelial cells in the adult lung. In contrast, CC10 expression is confined to Clara cells found in the airways of the adult mouse lung, and recombination mediated by the CC10-Cre line is restricted to these cells in the lung (21). Hopx is a transcriptional regulator that is expressed in lung epithelium after E13.5 and has recently been shown to act as a tumor suppressor in the lung (23, 24). Since we expressed both the KrasG12D and the Ctnnb1 $1^{\text {ex } 3 f l o x}$ oncogenes in Clara cells of the postnatal lung, we predicted that the resulting tumors would represent a bronchiolar airway phenotype. Surprisingly, tumors in CC10-Cre:Ctnnb1 ${ }^{\text {ex3flox: }}$ LSL-KrasG12D double mutant animals expressed high levels of SP-C and did not express CC10 or Hopx (Figure 2, C, F, and I). This is in contrast to CC10-Cre:LSL-KrasG12D single mutant tumors, which expressed a mix of SP-C- and CC10-positive cells and were Hopx positive (Figure 2, B, E, and H). Quantitative PCR (Q-PCR) confirmed the changes in SP-C and CC10 gene expression (Figure $2 \mathrm{M}$ ). These data suggested that tumors in CC10-Cre:Ctnnb1 $1^{\text {ex } 3 f l o x}: L S L$ KrasG12D double mutants represented a more immature epithelial phenotype similar to early distal or alveolar epithelium. Loss of Hopx may also contribute to the increased tumor development in

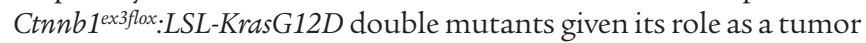
suppressor. Sca1 is also expressed in lung tumors from $L S L$-KrasG12D mutants (18). Sca1/CC10 double immunostaining showed that although the tumors in CC10-Cre:Ctnnb1 $1^{\text {ex } 3 f l o x}:$ LSL-KrasG12D double mutants did not express CC10, they did express Sca1 (Figure 2, J-L). Recent data have demonstrated that the bronchiolar epithelium represents a separate and distinct lineage that does not generate alveolar epithelium during homeostasis or after lung injury (25). Thus, these data suggest that coexpression of Ctnnb1 $1^{\text {ex3flox }}$ and KrasG12D in Clara cells of the postnatal lung results in a cell lineage switch from a bronchiolar lineage to an immature alveolar or distal epithelial lineage that resembles developing lung epithelium prior to E13.5.

Tumors observed in CC10-Cre:Ctnnb1 ${ }^{\text {ex } 3 f l o x}:$ LSL-KrasG12D mutants originate from Clara cells. Given the difference in tumor phenotype in CC10-Cre:Ctnnb1 $1^{\text {ex } 3 f l o x}:$ LSL-Kras G12D double mutants compared with their presumed cell of origin, we wanted to determine whether these tumors did indeed derive from Clara cells of the postnatal lung. Using the Rosa26R lacZ indicator line, we performed lacZ histochemical staining as well as $\beta$-galactosidase immunostaining of control, CC10-Cre:LSL-KrasG12D:R26R, and CC10-Cre:Ctnnb1 ex3flox:LSL-KrasG12D:R26R lungs. The data showed that the tumors in both CC10-Cre:LSL-KrasG12D:R26R and CC10Cre:Ctnnb1 ${ }^{\text {ex } 3 f l o x}: L S L-K r a s G 12 D: R 26 R$ mutants were derived from CC10-expressing Clara cells (Figure 3, A-F). Expression of SP-C on adjacent slides confirmed that the resulting tumors had altered their phenotype to a distal epithelial or immature alveolar lineage (Figure 3, G-I). Since CC10-expressing cells do not generate alveolar or distal epithelial cell types in the lung in lineage tracing experiments (25), these data are consistent with a cell lineage switch from CC10-positive bronchiolar epithelium to immature alveolar epithelium in CC10-Cre:Ctnnb1 ${ }^{\text {ex } 3 f l o x}:$ LSL-KrasG12D mutants. Alternatively, the previously identified bronchioalveolar stem cells (BASCs), which have been suggested to act as cancer stem cells, could also be the cancer-initiating cell of origin in these studies since they express CC10 (18).

Coexpression of Ctnnb1 $1^{\text {ex } 3 \text { flox }}$ and LSL-KrasG12D alleles leads to increased expression of markers of distal embryonic lung epithelium. To further determine the developmental state of the tumor epithelium generated by coactivation of KrasG12D and Ctnnb1 $1^{\text {ex } 3 f l o x}$, we performed immunostaining and in situ hybridization of multiple markers of both distal and proximal progenitors found in the embryonic lung. Tumors from CC10-Cre:Ctnnb1 $1^{\text {ex } 3 f l o x}: L S L$ KrasG12D double mutants expressed high levels of Sox9, Wnt7b, and Id2, which are expressed exclusively in embryonic distal endoderm progenitors within the developing lung (Figure 3, J-L and S-X, and refs. 26-31). CC10-Cre:Ctnnb1 ${ }^{\text {ex3flox }}$ :LSL-KrasG12D double mutant tumors also expressed high levels of Gata6, 
A
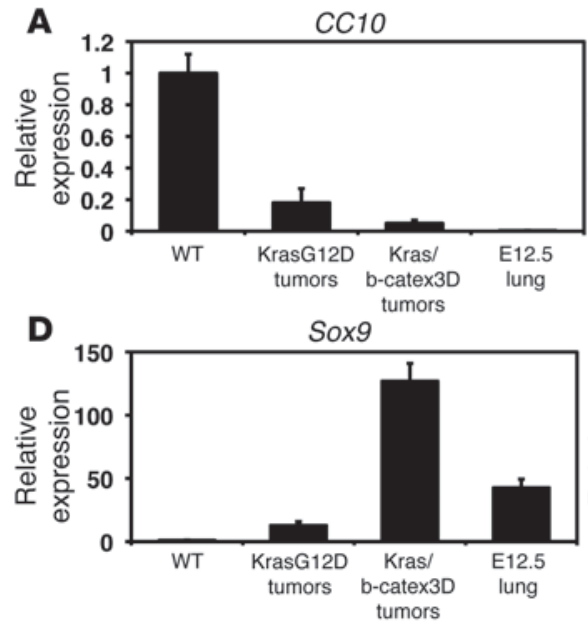

G

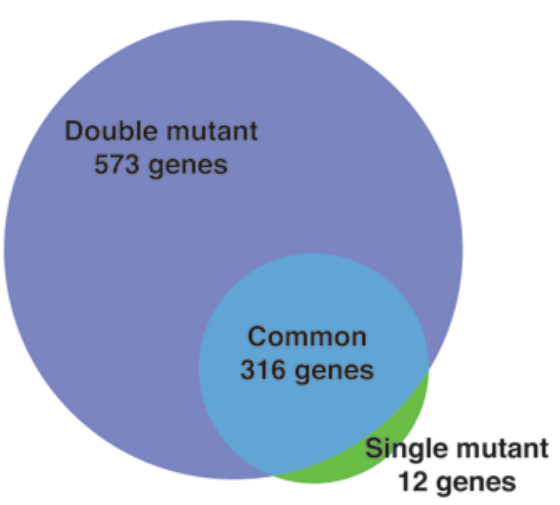

B

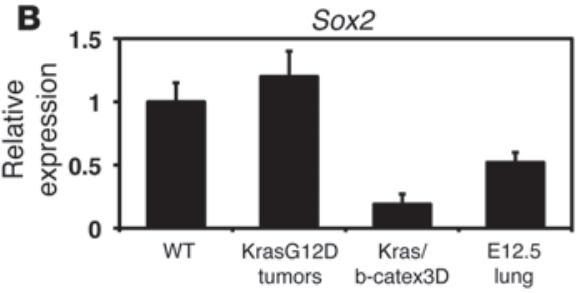

E

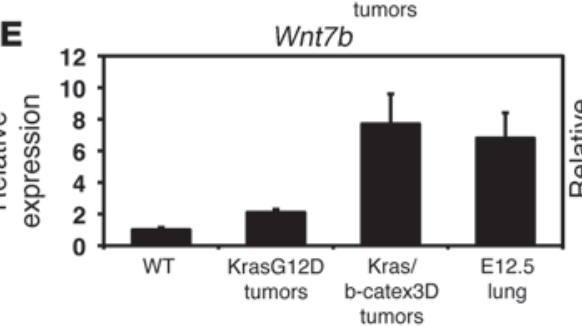

H

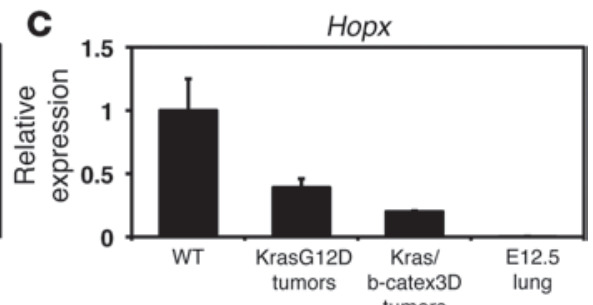

Gata6

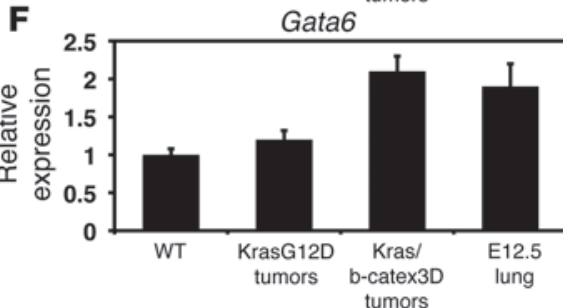

Biological process enrichment

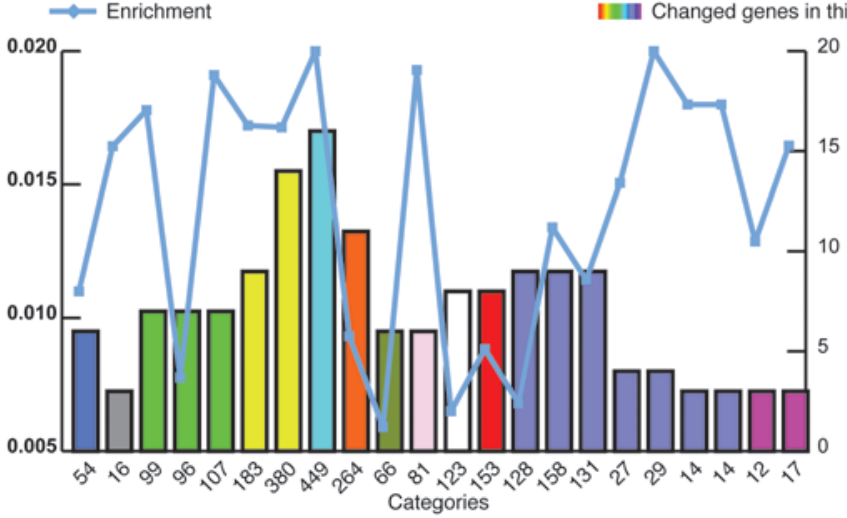

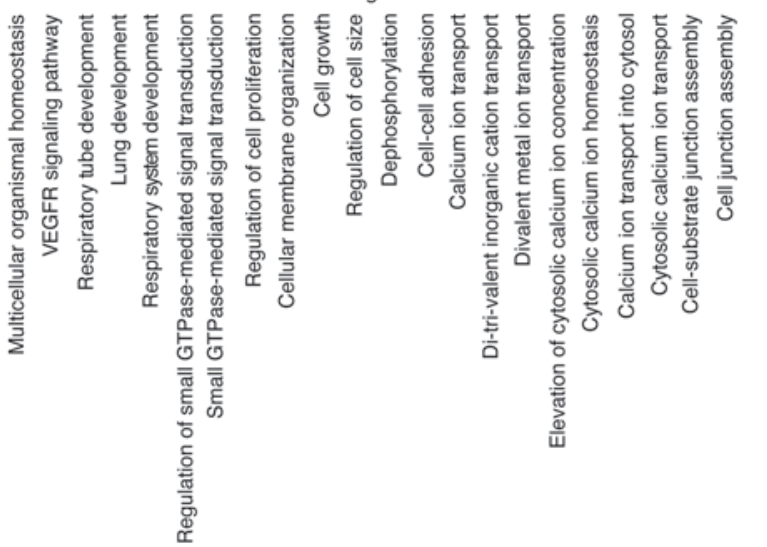

\section{Figure 4}

Comparison of CC10-Cre:KrasG12D and CC10-Cre:KrasG12D:Ctnnb1ex3flox tumor epithelium with embryonic lung epithelium and identification of molecular pathways altered in CC10-Cre:KrasG12D:Ctnnb1ex3flox lung epithelium. A comparison of CC10 (A), Sox2 (B), Hopx (C), Sox9 (D), Wnt7b (E), and Gata6 (F) expression in WT (CC10-Cre), CC10-Cre:KrasG12D, and CC10-Cre:KrasG12D:Ctnnb1ex3flox tumor epithelium. Three individual animals of the indicated genotypes were used for Q-PCR analysis shown in A-F, and data are presented as mean \pm SEM. Changes in gene expression for CC10 $(P<0.001)$, Sox2 $(P<0.01)$, Hopx $(P<0.003)$, Sox9 $(P<0.005)$, Wnt7b $(P<0.001)$, and Gata6 $(P<0.04)$ were considered significant as indicated. (G) Microarray analysis showing that 673 genes were uniquely altered in CC10-Cre:KrasG12D:Ctnnb1ex3tlox tumor epithelium. (H) Biological process enrichment graph showing molecular pathways altered in CC10-Cre:KrasG12D:Ctnnb1ex3flox tumor epithelium.

which is expressed at higher levels in distal progenitors than in more proximal progenitors during lung development (Figure 3, $\mathrm{P}-\mathrm{R}$, and refs. 32-35). However, Ctnnb1 ${ }^{\text {ex3flox }}$ :LSL-KrasG12D double mutant tumors did not express appreciable levels of Sox2, which is a marker of proximal epithelial progenitors in both the devel- oping and adult lung (Figure 3, M-O, and refs. 28, 36, 37). These changes in expression were confirmed by Q-PCR (Figure $3 Y$ ). In a direct comparison, CC10-Cre:Ctnnb1 ${ }^{\text {ex } 3 \text { flox }}$ :LSL-Kras G12D tumors were more similar to E12.5 lungs than adult lungs in their expression of Sox9, Wnt7b, Gata6, Hopx, CC10, and Sox2 (Figure 4, A-F). 


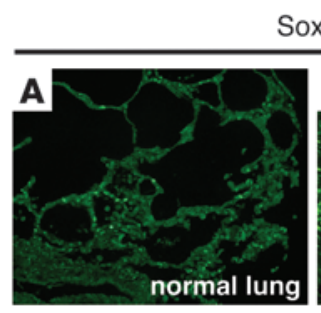

Sox9
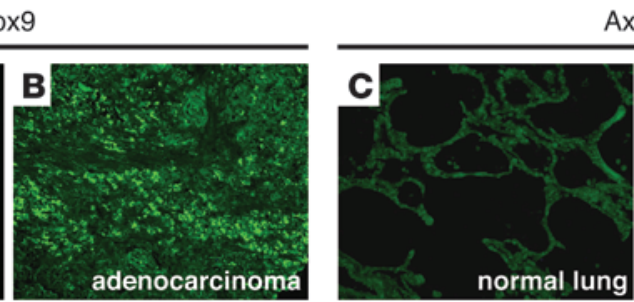

Axin2

E

Sox9-positive $62 \%$ of adenocarcinomas

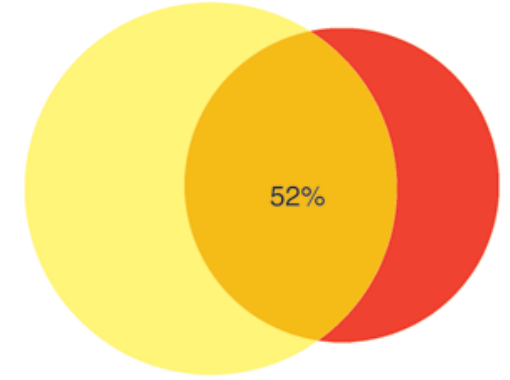

Axin2-positive $42 \%$ of adenocarcinomas
SOX9
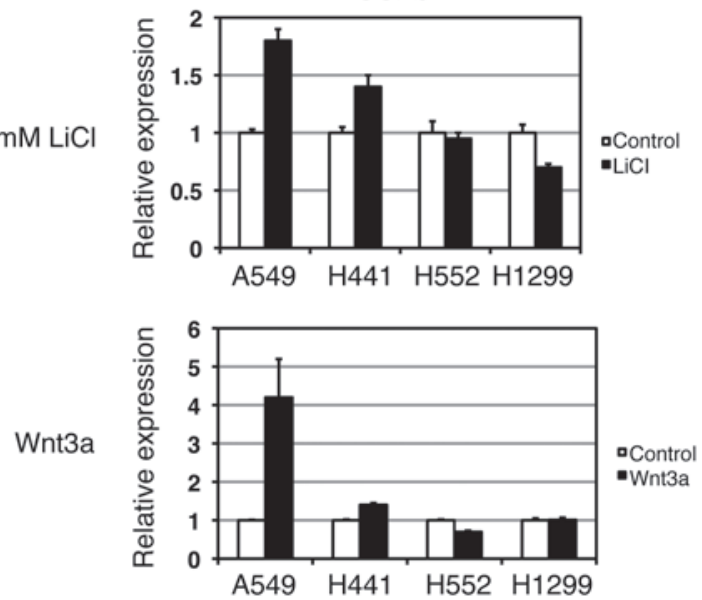

$\mathbf{F}$

$5 \mathrm{mM} \mathrm{LiCl}$

Wnt3a
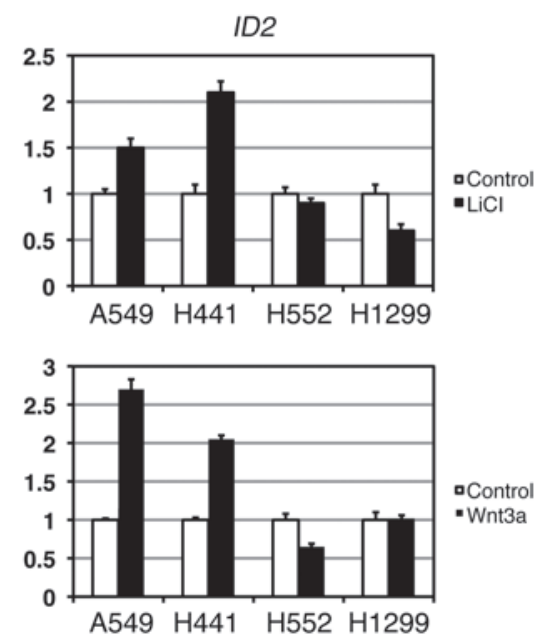

\section{Figure 5}

Sox9 expression in human lung adenocarcinomas. The human lung tumor array was immunostained for Sox9 and axin2 expression as described in Methods and scored by 3 independent researchers. Normal human lung contains only rare Sox9-positive cells (A) and almost no axin2-immunostaining cells (C). (B, D, and E) $62 \%$ of adenocarcinoma samples had high levels of Sox9, while $42 \%$ of adenocarcinoma samples were positive for axin2 immunoreactivity. The percentage of Sox9-positive samples that were axin2 positive was $52 \%(E)$. The samples used for Sox9 and Axin2 immunostaining were from the same normal lung and tumor samples. (F) $\mathrm{LiCl}$ and Wnt3a induce SOX9 and ID2 expression in $\mathrm{A} 549$ and $\mathrm{H} 441$ cells but not $\mathrm{H} 552$ and $\mathrm{H} 1299$ cells. Untreated cells served as controls in these assays. Original magnification, $\times 100$
The increased levels of Sox 9 and decreased levels of Sox2 in CC10-

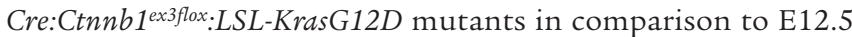
lung buds further supports a phenotypic change to a distal epithelial progenitor phenotype in these tumors.

Coactivation of Wnt/ß-catenin and oncogenic Kras signaling results in a distinct transcriptional profile representing an embryonic distal epithelial progenitor. The above data suggested that coactivation of oncogenic Kras and Wnt/ $\beta$-catenin signaling leads to a transdifferentiation of adult Clara cells to an embryonic distal progenitor phenotype. To further address this hypothesis, we performed microarray analysis of control, CC10-Cre:KrasG12D, and CC10-Cre:

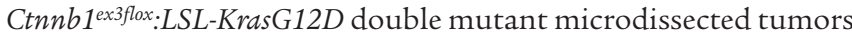
to determine their phenotype. From these studies we identified more than 600 genes whose deregulation was unique to the coexpression of Ctnnb1 $1^{\text {ex } 3 f l o x}$ and $L S L-K r a s G 12 D$ alleles (Figure $4 \mathrm{G}$ and Supplemental Table 1). Of these genes, several important molecular pathways were specifically affected in CC10-Cre:Ctnnb1 ${ }^{\text {ex3flox: }}$ LSL-KrasG12D double mutant tumors. As expected, GTP-mediated signal transduction and cell proliferation were significantly affected (Figure 4H). This analysis also showed increased enrichment of factors expressed during lung respiratory system development, during divalent cation transport, and in cell-cell adhesion (Figure $4 \mathrm{H}$ ). As predicted from the data above, important devel- opmental regulators such as $W n t 7 b, I d 2$, Sox 9 , and Mycn were all increased, whereas Hopx was decreased, in CC10-Cre:Ctnnb1 ${ }^{\text {ex3flox: }}$ LSL-Kras G12D tumors (Table 1).

To determine whether such embryonic regulators were also upregulated in human lung tumor samples, we performed Sox9 immunostaining on a human lung tumor array. This array consisted of histological sections from various phenotypically distinct tumor types including squamous cell carcinoma, small cell undifferentiated carcinoma, bronchioalveolar carcinoma, and adenocarcinoma. The majority (62\%) of adenocarcinomas were positive for Sox9 immunostaining, while normal lung tissue contained only rare Sox9-positive cells (Figure 5, A and B). Of note, Sox9 expression has been associated with prostate tumor development and basal cell carcinoma and is positively regulated by the Wnt/ $\beta$-catenin pathway (38-40). To determine whether these same tumor samples also expressed elevated levels of Wnt/ $\beta$-catenin signaling, we immunostained the same human lung tumor array to detect expression of axin2, a known target of Wnt signaling that can be used to measure signaling activity (41). Overall, $42 \%$ of all adenocarcinomas were positive for axin2 expression (Figure 5E). Moreover, $52 \%$ of the Sox 9 -positive samples were axin 2 positive, indicating a substantial correlation between Sox9 and increased Wnt/ $\beta$-catenin signaling activity (Figure $5 \mathrm{E}$ ). 

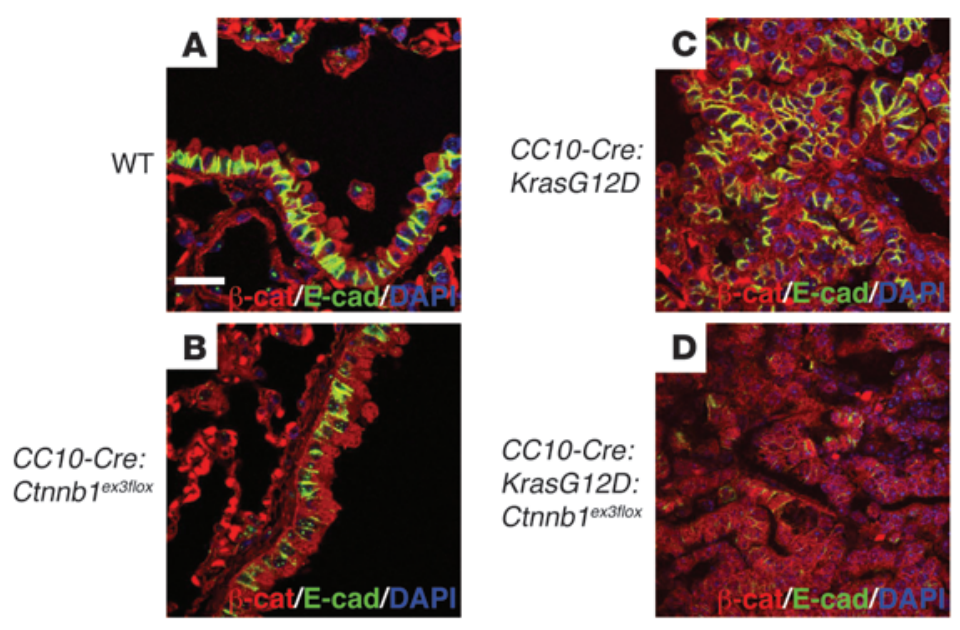

\section{E}

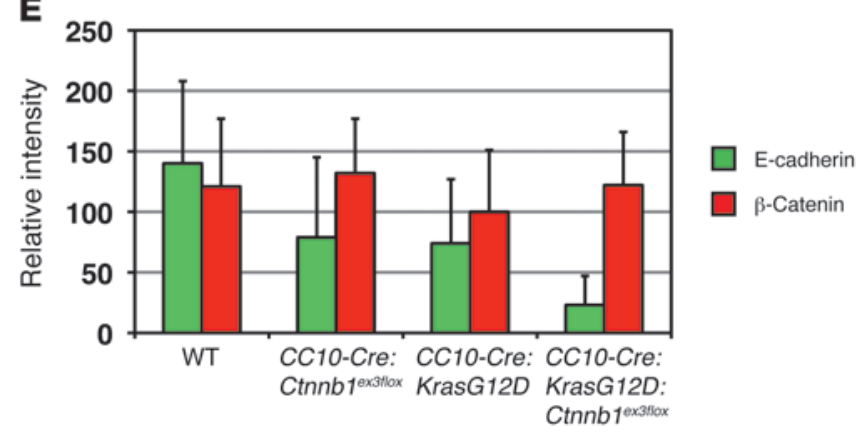

\section{Figure 6}

Loss of E-cadherin expression in CC10-Cre:KrasG12D:Ctnnb1ex3flox tumor epithelium. (A-D) Confocal microscopy showing loss of E-cadherin expression by immunostaining and z-axis scanning of confocal data. (E) E-cadherin expression quantified by relative intensity. Relative intensity changes were only significant for CC10-Cre:KrasG12D:Ctnnb1 ex3tlox tumor epithelium $(P<0.01)$. Scale bar: $50 \mu \mathrm{m}$.

To assess whether increased Wnt/ $\beta$-catenin signaling along with Kras signaling would increase expression of the distal progenitor marker genes SOX9 and ID2 in human lung cancer cell lines, we activated Wnt/ $\beta$-catenin signaling in two human lung tumor cell lines that harbor mutant forms of Kras, A549 and H441, and two cell lines that harbor wild-type Kras alleles, H552 and H1299 (42-44). Untreated cells served as negative controls in these assays. Activation of Wnt/ $\beta$-catenin signaling by Wnt3a and $\mathrm{LiCl}$, a known agonist of glycogen synthase kinase $3 \beta$ (GSK-3 $\beta$ ) and activator of Wnt signaling (45), resulted in increased expression of both Sox9 and Id 2 in A549 and H441 cells but not H552 or H1299 cells (Figure 5D). Together, these data support the imposition of a distal embryonic lung progenitor phenotype by coactivation of Wnt/ $\beta$-catenin and Kras signaling in both mouse and human lung epithelium.

Ctnnb $1^{\text {ex3flox: }}$ LSL-KrasG12D double mutant tumors exhibit decreased E-cadherin expression. A recent study showed that activation of Wnt/ $\beta$-catenin signaling in human lung tumor lines leads to increased metastatic potential (15). Disruption in cell-cell adhesion can lead to increased metastasis in cancer, and E-cadherin plays an important role in regulating this process in lung cancer (46-50). Moreover, alterations in $\beta$-catenin levels that occur upon Wnt activation can lead to changes in cadherin-catenin interactions and a loss of cadherin stability $(46,47,51,52)$. Therefore, we wanted to determine whether the integrity of cell-cell inter- actions, in particular E-cadherin-related complexes, was disrupted in CC10-Cre:Ctnnb1 ${ }^{\text {ex3flox }}:$ LSL-KrasG12D double mutant tumors. We used confocal microscopy to assess the expression of both E-cadherin and $\beta$-catenin in wildtype, CC10-Cre:Ctnnb1 $1^{\text {ex } 3 f l o x}$, CC10-Cre:LSL-KrasG12D, and CC10-Cre:Ctnnb1 ${ }^{\text {ex3flox}}:$ LSL-KrasG12D double mutant tumors. Although $\beta$-catenin was still present in the membrane of the compound mutant cells, expression of E-cadherin was markedly reduced at the cell surface as well as throughout the rest of the mutant epithelium (Figure 6 and Supplemental Figure 2). E-cadherin and $\beta$-catenin colocalization was also reduced in Ctnnb1 ${ }^{\text {ex } 3 f l o x}$ :LSL-KrasG12D double mutant tumors (Figure 6 and Supplemental Figure 2). These data suggest that loss of E-cadherin may underlie part of the increased metastatic potential of lung tumor cell lines with increased Wnt/ $\beta$-catenin signaling activation as previously reported, which is consistent with the function of $\mathrm{Wnt} / \beta$-catenin in other cancers such as pancreatic cancer (46-50). Despite the decreased E-cadherin expression in the Ctnnb1 ${ }^{\text {ex3flox:}}$ LSL-Kras G12D double mutant tumors, we did not observe metastasis in these animals, which is likely explained by the premature demise of the majority of the compound mutants by 4 months of age (data not shown).

\section{Discussion}

Our data show that Wnt/ $\beta$-catenin signaling can play a critical cooperative role with other oncogenic pathways such as Kras to promote lung tumorigenesis in part by altering the phenotype of the resulting tumor epithelium and promoting an embryonic distal progenitor phenotype (Figure 7). Such a change results in an accelerated growth rate and may lead to greater metastasis due to decreased E-cadherin expression, as has been previously suggested (15). Thus, Wnt/ $\beta$-catenin signaling acts synergistically to promote lung tumorigenesis, and screening for activation of the pathway may be important in the phenotyping of human lung tumor samples.

Wnt/ $\beta$-catenin signaling has been long known to cause tumorigenesis in several tissues, most notably colon and skin $(8,20)$. Its role in other epithelial cancers has been less clear, and in lung cancer, mutations in the $\mathrm{Wnt} / \beta$-catenin pathway are not found at high frequency $(10,11,13,14)$. The cell of origin for most lung cancers remains elusive, but several studies have pointed to cells residing in the BADJ region $(18,53)$. As with these other studies, we observed increased incidence of tumor formation in this region, especially in the Kras activation model $(18,54)$. Although it was difficult to determine whether this also occurred in the Ctnnb1 $1^{\text {ex3flox }}$ :LSL-KrasG12D double mutant model, since these tumors developed more rapidly and were significantly larger, based on our use of the CC10-Cre line to activate both pathways, our data suggest that the origin of these tumors is either BASC-like cells or Clara cells within the bronchiolar epithelium. The lack of increased proliferation or tumor formation upon activation of Wnt/ $\beta$-catenin signaling in bronchiolar epithelium in the present study as well as previous studies is somewhat unexpected and suggests that these cells are relatively resistant to oncogenic transformation by this pathway (12).

The finding that coactivation of both oncogenic Kras and Wnt/ $\beta$-catenin leads to a transdifferentiation of Clara cells to a distal embryonic progenitor phenotype is interesting in light of what is 


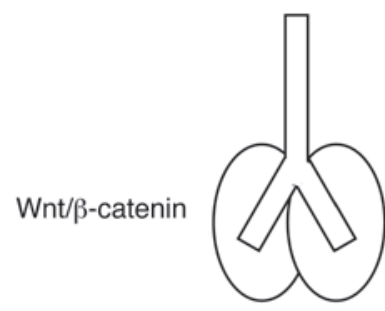

Normal-no tumor development

Tumor development-adenomas

Tumor cells maintain

bronchiolar phenotype

KrasG12D

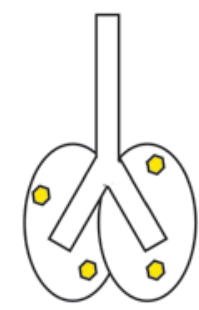

Advanced tumor development-adenocarcinoma

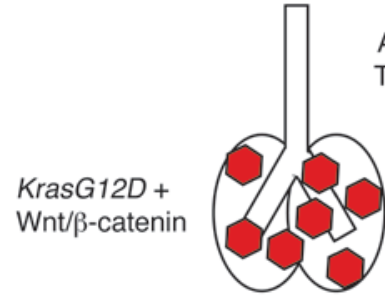

Transdifferentiation to distal embryonic progenitor

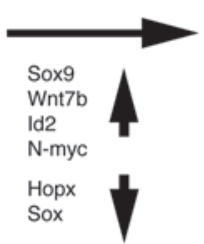

Decreased E-cadherin

Increased metastasis?

\section{Figure 7}

Model of $\mathrm{Wnt} / \beta$-catenin and oncogenic Kras interactions and the resulting alterations in airway epithelial phenotype leading to an acceleration of lung tumor development.

known about Wnt $/ \beta$-catenin signaling in development. Wnt $/ \beta$ catenin signaling plays multiple roles in lung epithelial development, starting with a requirement of this pathway to specify Nkx2.1positive lung endoderm progenitors within the anterior foregut (55). After specification, loss of Wnt/ $\beta$-catenin signaling leads to a loss of distal epithelial progenitors and subsequent distal epithelial and alveolar differentiation $(56,57)$. Conversely, forced activation of Wnt $/ \beta$-catenin signaling throughout the anterior foregut endoderm in early development leads to the expansion of Nkx2.1-positive lung endoderm progenitors into the esophagus and stomach, suggesting a dominant role in lung progenitor specification (55). The imposition of a distal embryonic progenitor phenotype on lung tumors derived from Clara cells is supported by these findings and suggests that Wnt/ $\beta$-catenin signaling can transdifferentiate bronchiolar epithelium under certain conditions, including coactivation of oncogenic Kras. This is similar to what is suggested to occur in the intestine, where Wnt/ $\beta$-catenin imposes a crypt progenitor phenotype when activated in differentiated intestinal epithelium (58). The decrease in expression of the lung tumor suppressor gene Hopx and increased expression of Mycn also point to a cellular phenotype that is predisposed to oncogenic development. Given the highly proliferative and relatively undifferentiated state of embryonic distal lung progenitors, it will be important to further explore pathways such as Wnt/ $\beta$-catenin that promote this phenotype in the context of new anticancer therapies as well as prognostic assays in humans.

Our data suggest that instead of assessing for mutations in Wnt/ $\beta$-catenin pathway components as a primary cause of lung tumorigenesis, a better rationale may be to determine whether such muta- tions are associated with a more aggressive form of lung cancer. Previous work has shown a positive correlation with TCF4 target gene activation and primary pulmonary tumor metastasis (15). These studies showed that increased $\mathrm{Wnt} / \beta$-catenin signaling in human lung tumor cell lines increased their metastatic potential. However, why these cells were more metastatic was unclear. Our data suggest that the downregulation of E-cadherin, which is known to be associated with increased metastasis in lung cancer as well as other cancers including pancreatic cancer, may be an important mechanism by which this increased metastasis occurs $(46-48,50)$. Cadherin-catenin complexes play important roles in the stabilization of cell-cell junctions in many types of epithelia. The interactions between $\beta$-catenin and $\mathrm{E}$-cadherin are regulated by multiple pathways including Wnt signaling. The loss of E-cadherin in the present model may be due to the accumulation of $\beta$-catenin in the nucleus and a reduction in the adherens junctions leading to decreased stability and ultimately degradation of E-cadherin. This loss of adherens junction stability could lead to increased lung tumor epithelial metastasis, and such associations have been described previously $(46,47)$. It is interesting to note that neither increased Wnt signaling alone nor expression of oncogenic Kras caused an appreciable decrease in E-cadherin. These data suggest that the alteration in multiple oncogenic pathways is required to disrupt cell-cell junction stability and promote metastasis in the bronchiolar epithelium of the lung. This is in line with what is observed in human lung cancer cell lines, where Wnt signaling promotes a metastatic phenotype in cells that have already undergone an oncogenic transformation (15). However, we did not observe metastasis in our model, which is likely due to the premature lethality observed in the compound mutants. Our data indicate that Wnt/ $\beta$-catenin activation in combination with Kras mutations leads to a more aggressive form of the disease with an embryonic progenitor phenotype. These studies may lead to a better understanding of human lung cancer and development of additional diagnostic assays to help define the heterogeneity in human lung cancer phenotypes.

\section{Methods}

Animals. The generation and genotyping of Ctnnb1 $1^{e x 3 f l o x}$, CC10-Cre, $L S L-K r a s G 12 D$, and $R 26 R$ mice has been previously described (20, 21, 54, 59). Three- to 4-month-old mice were used for the experiments unless otherwise specified. Animal experiments were approved by the University of Pennsylvania Institutional Animal Care and Use Committee and conformed to the relevant regulatory standards.

Histology. Lungs were inflated and fixed with $2 \%$ paraformaldehyde overnight, then gradually dehydrated in ethanol before being subjected to paraffin embedding for sectioning. In situ hybridization was performed for Hopx, Gata6, and Wnt7b as previously described $(24,27,32)$. Immunohistochemistry (IHC) and whole mount lacZ histochemical staining were performed as previously described (27), and detailed protocols are available at http://www.med.upenn.edu/mcrc/histology_core/. Antibodies were used at the indicated dilutions: CC10 1:20 (Santa Cruz Biotechnology Inc., sc-9772), Ki-67 1:50 (Dako, M-7249), TTF-1 1:50 (Santa Cruz Biotechnology Inc., sc-13040), SP-C 1:500 (Chemicon, AB3786), SP-B 1:100 (Chemicon, AB3780), Sca1 1:50 (R\&D Systems, MAB1226), $\beta$-galactosidase 
1:250 (MP Biomedicals, 55976), Sox9 1:100 (Santa Cruz Biotechnology Inc., sc-20095), Sox2 1:100 (Chemicon, AB5603), Id2 1:25 (CalBioreagents, M213), $\beta$-catenin 1:100 (BD Biosciences, 610154), E-cadherin 1:100 (Cell Signaling Technology, 3195), and axin2 1:100 (Abcam, 32197). All histological procedures were performed on at least 4 animals of the indicated genotype, and representative data are shown.

Human lung tumor tissue array. Sox9 expression in human lung tumor samples was assessed using a human tissue array consisting of 120 distinct human lung tumor samples (48 adenocarcinomas) along with normal adult lung tissue (catalog BC041115, US Biomax Inc.) and the anti-Sox9 and -axin 2 antibodies listed above. Sox 9 and axin 2 positivity was blindly scored by 3 independent researchers, and the data represent the samples that were scored positive by all 3 individuals.

Cell culture analysis. A549, H441, H552, and H1299 cells were cultured as described previously (42-44). $\mathrm{LiCl}(5 \mathrm{mM})$ or recombinant Wnt3a $(100 \mathrm{ng} / \mathrm{ml})$ was added to the culture medium for 72 hours prior to harvesting of cells for RNA extraction.

Microscopy. Images were captured using a Nikon Eclipse 80i microscope with Nikon DXM1200F digital camera and Nikon ACT-1 version 2.63 software. Confocal images were captured using a Zeiss Axiovert 100 inverted microscope and were analyzed using Volocity 5 software (PerkinElmer) and Image (http://rsbweb.nih.gov/ij/).

Microarray and PCR analysis. Lungs of 4-month-old mice were PBS inflated, tumors were dissected, and TRIzol was used to isolate RNA for both PCR and microarray analysis. For Q-PCR analysis, RNA was reverse transcribed to generate CDNA, which was used in real-time PCR analysis of gene expression. The oligonucleotide primers used are listed in Supplemental
Table 2. For microarray experiments, Affymetrix Mouse Gene 1.0 ST arrays were used. Microarray data were analyzed using robust multichip analysis (RMA) and principal component analysis (PCA) and Partek Genomics Suite version 6.5 . The algorithm used to select transcripts with significant changes in gene expression was a less than $5 \%$ false discovery rate $(P<0.05)$ and more than 2 -fold difference in the double mutant compared with the wild-type and a less than 1.75-fold difference between single mutant (CC10Cre:Kras G12D) and wild-type and for the single mutant tumors. Gene lists were tested for enrichment using High Throughput GoMiner software available from http://discover.nci.nih.gov/gominer/citing.jsp. Microarray data have been deposited in the GEO database (GSE26850).

Statistics. Two-tailed Student's $t$ test was used to assess significance of changes in relative expression using Q-PCR, and the values are indicted in each figure. $P$ values less than 0.05 were considered significant.

\section{Acknowledgments}

These studies were supported by funding from the NIH (HL087825 and HL064632 to E.E. Morrisey). We thank Brian Keith and Celeste Simon for their helpful comments on the manuscript.

Received for publication August 23, 2010, and accepted in revised form February 2, 2011.

Address correspondence to: Edward E. Morrisey, University of Pennsylvania, 956 BRB II/III, 421 Curie Blvd., Philadelphia, Pennsylvania, USA. Phone: 215.573.3010; Fax: 215.573.2094; E-mail: emorrise@mail.med.upenn.edu.
1. Jemal A, Siegel R, Ward E, Hao Y, Xu J, Thun MJ. Cancer statistics, 2009. CA Cancer J Clin. 2009;59(4):225-249.

2. Edwards BK, et al. Annual report to the nation on the status of cancer, 1975-2006, featuring colorectal cancer trends and impact of interventions (risk factors, screening, and treatment) to reduce future rates. Cancer. 2010;116(3):544-573.

3. Pao W, et al. EGF receptor gene mutations are common in lung cancers from "never smokers" and are associated with sensitivity of tumors to gefitinib and erlotinib. Proc Natl Acad Sci U S A. 2004;101(36):13306-13311.

4. Paez JG, et al. EGFR mutations in lung cancer: correlation with clinical response to gefitinib therapy. Science. 2004;304(5676):1497-1500.

5. Lynch TJ, et al. Activating mutations in the epidermal growth factor receptor underlying responsiveness of non-small-cell lung cancer to gefitinib. NEngl J Med. 2004;350(21):2129-2139.

6. Mills NE, Fishman CL, Rom WN, Dubin N, Jacobson DR. Increased prevalence of K-ras oncogene mutations in lung adenocarcinoma. Cancer Res. 1995; 55(7):1444-1447.

7. Rodenhuis S, Slebos RJ. The ras oncogenes in human lung cancer. Am Rev Respir Dis. 1990; 142(6 pt 2):S27-S30.

8. Chan EF, Gat U, McNiff JM, Fuchs E. A common human skin tumour is caused by activating mutations in beta-catenin. Nat Genet. 1999; 21(4):410-413.

9. Fodde R, Brabletz T. Wnt/beta-catenin signaling in cancer stemness and malignant behavior. Curr Opin Cell Biol. 2007;19(2):150-158.

10. Ding L, et al. Somatic mutations affect key pathways in lung adenocarcinoma. Nature. 2008; 455(7216):1069-1075.

11. Ohgaki H, Kros JM, Okamoto Y, Gaspert A, Huang $\mathrm{H}$, Kurrer MO. APC mutations are infrequent but present in human lung cancer. Cancer Lett. 2004; 207(2):197-203.

12. Reynolds SD, et al. Conditional stabilization of beta-catenin expands the pool of lung stem cells. Stem Cells. 2008;26(5):1337-1346.

13. Shigemitsu K, et al. Genetic alteration of the betacatenin gene (CTNNB1) in human lung cancer and malignant mesothelioma and identification of a new 3p21.3 homozygous deletion. Oncogene. 2001;20(31):4249-4257.

14. Sunaga N, Kohno T, Kolligs FT, Fearon ER, Saito R, Yokota J. Constitutive activation of the Wnt signaling pathway by CTNNB1 (beta-catenin) mutations in a subset of human lung adenocarcinoma. Genes Chromosomes Cancer. 2001;30(3):316-321.

15. Nguyen DX, et al. WNT/TCF signaling through LEF1 and HOXB9 mediates lung adenocarcinoma metastasis. Cell. 2009;138(1):51-62.

16. Rubin EM, Guo Y, Tu K, Xie J, Zi X, Hoang BH. Wnt inhibitory factor 1 decreases tumorigenesis and metastasis in osteosarcoma. Mol Cancer Ther. 2010;9(3):731-741.

17. DiMeo TA, et al. A novel lung metastasis signature links Wnt signaling with cancer cell self-renewal and epithelial-mesenchymal transition in basal-like breast cancer. Cancer Res. 2009;69(13):5364-5373.

18. Kim CF, et al. Identification of bronchioalveolar stem cells in normal lung and lung cancer. Cell. 2005;121(6):823-835.

19. Meylan E, et al. Requirement for NF-kappaB signalling in a mouse model of lung adenocarcinoma. Nature. 2009;462(7269):104-107.

20. Harada $\mathrm{N}$, et al. Intestinal polyposis in mice with a dominant stable mutation of the beta-catenin gene. EMBO J. 1999;18(21):5931-5942.

21. Li H, Cho SN, Evans CM, Dickey BF, Jeong JW, DeMayo FJ. Cre-mediated recombination in mouse Clara cells. Genesis. 2008;46(6):300-307.

22. Nikitin AY, et al. Classification of proliferative pulmonary lesions of the mouse: recommendations of the mouse models of human cancers consortium. Cancer Res. 2004;64(7):2307-2316.

23. Chen Y, Pacyna-Gengelbach M, Deutschmann N, Niesporek S, Petersen I. Homeobox gene HOP has a potential tumor suppressive activity in human lung cancer. Int J Cancer. 2007;121(5):1021-1027.

24. Yin Z, et al. Hop functions downstream of $\mathrm{Nkx} 2.1$ and GATA6 to mediate HDAC-dependent negative regulation of pulmonary gene expression. Am J Physiol Lung Cell Mol Physiol. 2006;291(2):L191-L199.

25. Rawlins EL, et al. The role of Scgb1a1+ Clara cells in the long-term maintenance and repair of lung airway, but not alveolar, epithelium. Cell Stem Cell. 2009;4(6):525-534.

26. Jen Y, Manova K, Benezra R. Expression patterns of Id1, Id2, and Id 3 are highly related but distinct from that of Id 4 during mouse embryogenesis. Dev Dyn. 1996;207(3):235-252.

27. Shu W, Jiang YQ, Lu MM, Morrisey EE. Wnt7b regulates mesenchymal proliferation and vascular development in the lung. Development. 2002;129(20):4831-4842.

28. Okubo T, Knoepfler PS, Eisenman RN, Hogan BL. Nmyc plays an essential role during lung development as a dosage-sensitive regulator of progenitor cell proliferation and differentiation. Development. 2005;132(6):1363-1374.

29. Perl AK, Kist R, Shan Z, Scherer G, Whitsett JA. Normal lung development and function after Sox 9 inactivation in the respiratory epithelium. Genesis. 2005;41(1):23-32.

30. Lu Y, Thomson JM, Wong HY, Hammond SM, Hogan BL. Transgenic over-expression of the microRNA miR-17-92 cluster promotes proliferation and inhibits differentiation of lung epithelial progenitor cells. Dev Biol. 2007;310(2):442-453.

31. Rawlins EL, Clark CP, Xue Y, Hogan BL. The Id2+ distal tip lung epithelium contains individual multipotent embryonic progenitor cells. Development. 2009;136(22):3741-3745.

32. Morrisey EE, Ip HS, Lu MM, Parmacek MS. GATA6: a zinc finger transcription factor that is expressed in multiple cell lineages derived from lateral mesoderm. Dev Biol. 1996;177(1):309-322.

33. Yang H, Lu MM, Zhang L, Whitsett JA, Morrisey EE. GATA6 regulates differentiation of distal lung epithelium. Development. 2002;129(9):2233-2246. 
34. Zhang Y, et al. GATA and Nkx factors synergistically regulate tissue-specific gene expression and development in vivo. Development. 2007;134(1):189-198.

35. Zhang Y, et al. A Gata6-Wnt pathway required for epithelial stem cell development and airway regeneration. Nat Genet. 2008;40(7):862-870.

36. Que J, et al. Multiple dose-dependent roles for Sox 2 in the patterning and differentiation of anterior foregut endoderm. Development. 2007;134(13):2521-2531.

37. Que J, Luo X, Schwartz RJ, Hogan BL. Multiple roles for Sox 2 in the developing and adult mouse trachea. Development. 2009;136(11):1899-1907.

38. Blache $\mathrm{P}$, et al. SOX9 is an intestine crypt transcription factor, is regulated by the Wnt pathway, and represses the CDX2 and MUC2 genes. J Cell Biol. 2004;166(1):37-47.

39. Vidal VP, Ortonne N, Schedl A. SOX9 expression is a general marker of basal cell carcinoma and adnexal-related neoplasms. J Cutan Pathol. 2008; 35(4):373-379.

40. Thomsen MK, et al. SOX9 elevation in the prostate promotes proliferation and cooperates with PTEN loss to drive tumor formation. Cancer Res. 2010; 70(3):979-987.

41. Jho EH, Zhang T, Domon C, Joo CK, Freund JN, Costantini F. Wnt/beta-catenin/Tcf signaling induces the transcription of Axin2, a negative regulator of the signaling pathway. Mol Cell Biol. 2002; 22(4):1172-1183

42. van Haaften $G$, et al. Somatic mutations of the histone H3K27 demethylase gene UTX in human cancer. Nat Genet. 2009;41(5):521-523.

43. Lopez-Crapez E, Chypre C, Saavedra J, Marchand J, Grenier J. Rapid and large-scale method to detect $\mathrm{K}$-ras gene mutations in tumor samples. Clin Chem. 1997;43(6 pt 1):936-942.

44. Davies $\mathrm{H}$, et al. Mutations of the BRAF gene in human cancer. Nature. 2002;417(6892):949-954.

45. Klein PS, Melton DA. A molecular mechanism for the effect of lithium on development. Proc Natl Acad Sci U S A. 1996;93(16):8455-8459.

46. Asnaghi L, et al. E-cadherin negatively regulates neoplastic growth in non-small cell lung cancer: role of Rho GTPases. Oncogene. 2010;29(19):2760-2771.

47. Bremnes RM, Veve R, Hirsch FR, Franklin WA. The E-cadherin cell-cell adhesion complex and lung cancer invasion, metastasis, and prognosis. Lung Cancer. 2002;36(2):115-124.

48. Myong NH. Reduced expression of E-cadherin in human non-small cell lung carcinoma. Cancer Res Treat. 2004;36(1):56-61.

49. Saad AG, et al. Immunohistochemical markers associated with brain metastases in patients with nonsmall cell lung carcinoma. Cancer. 2008; 113(8):2129-2138.

50. von Burstin J, et al. E-cadherin regulates metastasis of pancreatic cancer in vivo and is suppressed by a SNAIL/HDAC1/HDAC2 repressor complex. Gastroenterology. 2009;137(1):361-371.

51. Ma L, et al. miR-9, a MYC/MYCN-activated micro-
RNA, regulates E-cadherin and cancer metastasis. Nat Cell Biol. 2010;12(3):247-256.

52. Wang XQ, Li H, Van Putten V, Winn RA, Heasley LE, Nemenoff RA. Oncogenic K-Ras regulates proliferation and cell junctions in lung epithelial cells through induction of cyclooxygenase- 2 and activation of metalloproteinase-9. Mol Biol Cell. 2009;20(3):791-800.

53. Kim CF, et al. Mouse models of human non-smallcell lung cancer: raising the bar. Cold Spring Harb Symp Quant Biol. 2005;70:241-250.

54. Jackson EL, et al. Analysis of lung tumor initiation and progression using conditional expression of oncogenic K-ras. Genes Dev. 2001;15(24):3243-3248.

55. Goss AM, et al. Wnt $2 / 2 \mathrm{~b}$ and beta-catenin signaling are necessary and sufficient to specify lung progenitors in the foregut. Dev Cell. 2009;17(2):290-298.

56. Mucenski ML, et al. beta-Catenin is required for specification of proximal/distal cell fate during lung morphogenesis. J Biol Chem. 2003;278(41):40231-40238.

57. Shu W, et al. Wnt/beta-catenin signaling acts upstream of $\mathrm{N}-\mathrm{myc}, \mathrm{BMP} 4$, and FGF signaling to regulate proximal-distal patterning in the lung. Dev Biol. 2005;283(1):226-239.

58. van de Wetering M, et al. The beta-catenin/TCF-4 complex imposes a crypt progenitor phenotype on colorectal cancer cells. Cell. 2002;111(2):241-250.

59. Soriano P. Generalized lacZ expression with the ROSA26 Cre reporter strain. Nat Genet. 1999;21(1):70-71. 